\section{E-194 PATIENT-SPECIFIC HEMODYNAMICS PREDICT ENDOVASCULAR COILING OUTCOMES FOR INTRACRANIAL ANEURYSMS}

1,2 Levy* ${ }^{3} \mathrm{~L}$ Marsh, ${ }^{3} \mathrm{M}$ Barbour, ${ }^{1,2} \mathrm{~K}$ Prijoles, ${ }^{1,2} \mathrm{D}$ Lim, ${ }^{1,2} \mathrm{H}$ Haughn, ${ }^{1,2} \mathrm{C}$ Kelly, ${ }^{3} \mathrm{~F}$ Chassange, ${ }^{1} \mathrm{G}$ Barros, ${ }^{1} \mathrm{D}$ Bass, ${ }^{3,2} \mathrm{~A}$ Aliseda, ${ }^{1,2} \mathrm{M}$ Levitt, ${ }^{1,2} \mathrm{~L} \mathrm{Kim} .{ }^{1}$ Neurological Surgery, University of Washington School of Medicine, Seattle, WA; ${ }^{2}$ Stroke and Applied Neuroscience Center, Seattle, WA; ${ }^{3}$ Mechanical Engineering, University of Washington, Seattle, WA

\subsection{6/neurintsurg-2020-SNIS.225}

Introduction Embolic coils are a preferred endovascular treatment technique for intracranial aneurysms, but approximately $34 \%$ of these treatments are unsuccessful, requiring re-treatment. To better understand why endovascular treatment fails, we used computational fluid dynamics (CFD) simulations to determine the changes in hemodynamics factors after coil embolization that were associated with treatment success versus those that led to failure. We incorporated patient-specific intravascular blood flow measurements as boundary conditions, which has been shown to improve simulation accuracy.

Materials and Methods Adult patients presenting with unruptured intracranial aneurysms treated with coil embolization were enrolled. 3D-rotational angiograms taken before and immediately after treatment were used to create computational models of the patients' vasculature. Intraoperative blood velocity and blood pressure measurements were obtained before and immediately after coil embolization by placing a dual-sensor microwire (ComboWire) in the proximal segment of the parent vessel. These measurements were incorporated as patient-specific boundary conditions for the CFD simulations to compute hemodynamics conditions before and after treatment. Hemodynamics variables including inflow rate (Q), wall shear stress (WSS), and wall shear stress gradient (WSSG) were calculated within each aneurysm. Treatment success or failure was determined by evaluating each patient's most recent follow-up angiogram, and this outcome was then correlated to the changes in hemodynamics

\begin{tabular}{|c|c|c|c|c|c|c|}
\hline$N$ & Age & Sex & $\begin{array}{l}\mathrm{Hx} \\
\text { Smoking }\end{array}$ & $\begin{array}{l}\text { Hx } \\
\text { HTN }\end{array}$ & $\begin{array}{l}\text { Aneurysm } \\
\text { Location }\end{array}$ & $\begin{array}{l}\text { Aneurysm Size } \\
(\mathrm{mm})\end{array}$ \\
\hline 1 & 36 & $\mathrm{~F}$ & No & No & R ICA cavernous & 5.6 \\
\hline 2 & 73 & $\mathrm{~F}$ & Yes & Yes & L ICA ophthalmic & 10.0 \\
\hline 3 & 48 & M & No & Yes & L ICA clinoid & 4.5 \\
\hline 4 & 74 & $\mathrm{~F}$ & Yes & Yes & L ICA PCOM & 11.5 \\
\hline 5 & 47 & M & Yes & No & BA R AICA & 6.5 \\
\hline 6 & 60 & $M$ & Yes & No & BA terminus & 11.0 \\
\hline 7 & 50 & $\mathrm{~F}$ & Yes & No & L ICA ophthalmic & 5.2 \\
\hline 8 & 58 & $\mathrm{~F}$ & No & Yes & BA terminus & 6.0 \\
\hline 9 & 61 & M & No & No & L ICA opthalamic & 5.3 \\
\hline 10 & 69 & $\mathrm{~F}$ & Yes & No & BA L SCA & 5.8 \\
\hline 11 & 56 & $\mathrm{~F}$ & Yes & Yes & R ICA ophthalmic & 11.8 \\
\hline 12 & 40 & $\mathrm{~F}$ & No & No & L ICA PCOM & 8.5 \\
\hline 13 & 44 & $\mathrm{~F}$ & No & No & L ICA clinoid & 5.8 \\
\hline 14 & 69 & $M$ & Yes & Yes & R ICA terminus & 5.6 \\
\hline 15 & 55 & $\mathrm{~F}$ & Yes & Yes & L ICA ophthalmic & 5.8 \\
\hline 16 & 52 & $\mathrm{~F}$ & No & Yes & L ICA opthalmic & 6.4 \\
\hline
\end{tabular}

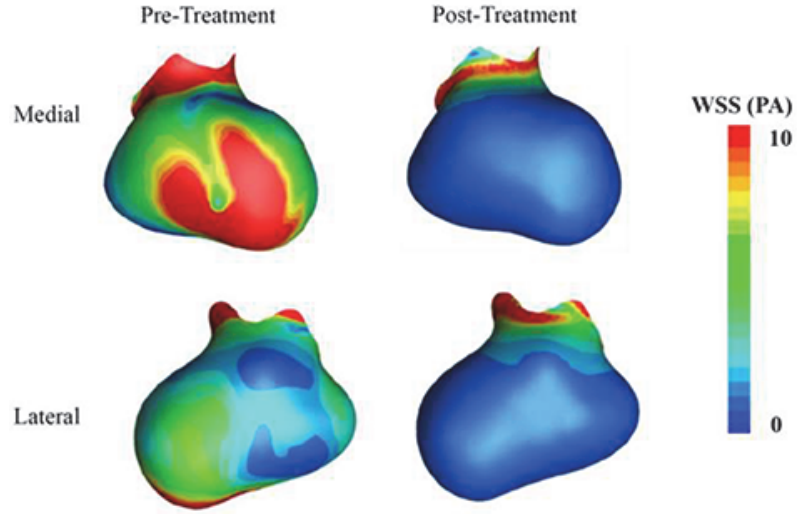

Abstract E-194 Figure 1 Patient-specific computational fluid dynamics modelling (CFD) of changes in wall shear stress (WSS) in an

variables pre- and post-treatment. This statistical analysis aims to determine associations between immediate changes in post-treatment hemodynamics variables and treatment outcomes.

Results Sixteen patients were included (table 1). Regardless of long-term treatment outcome, immediate post-treatment reductions were observed in Q, WSS and WSSG. Preliminary analyses suggest that an increase in shear stress at the aneurysm neck was associated with successful long-term outcome, representative of the significant redirection of blood flow velocity by the coil mass required to prevent aneurysm recanalization. Final multivariate analyses will be presented.

Conclusion CFD simulations of aneurysm hemodynamics using patient-specific imaging and boundary conditions were performed in a large cohort of patients treated with coil embolization. Increases in neck plane shear and redirection of blood flow found immediately post-coil embolization have the potential to predict long-term treatment success.

Disclosures S. Levy: None. L. Marsh: None. M. Barbour: None. K. Prijoles: None. D. Lim: None. H. Haughn: None. C. Kelly: None. F. Chassange: None. G. Barros: None. D. Bass: None. A. Aliseda: None. M. Levitt: 1; C; Philips Volcano. L. Kim: None.

\section{E-195 A MIXED REALITY SPATIAL COMPUTING FRAMEWORK FOR PREPROCEDURAL EVALUATION OF CEREBRAL ANEURYSMS: APPROACH AND PRELIMINARY RESULTS}

${ }^{1} G$ Deib*, ${ }^{2} D$ Smith, ${ }^{2} B$ Chaudhary, 'S Boo, ${ }^{1} A$ Tarabishy, ${ }^{1} \mathrm{~J}$ Carpenter, ${ }^{1} A$ Rai. ${ }^{1}$ Neuroradiology, West Virginia University, Morgantown, WV; ${ }^{2}$ Reed College of Media, West Virginia University, Morgantown, WV

\subsection{6/neurintsurg-2020-SNIS.226}

Introduction/Purpose Mixed Reality (MxR) is an emerging technology that makes seamless connections between virtual space and the real world by superimposing computer-generated information onto the real-world environment. MxR can provide additional information in a more intuitive and natural way than alternative information-delivery methods 


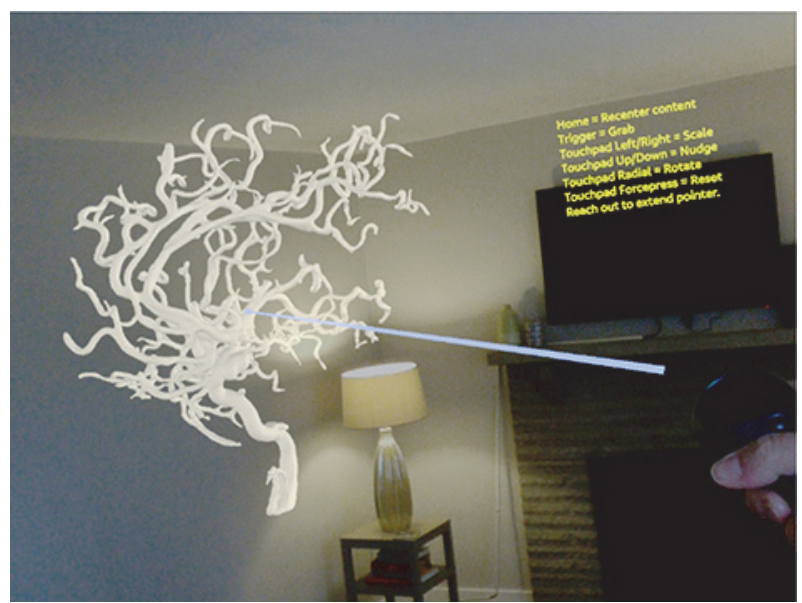

Abstract E-195 Figure 1

such as traditional angiographic monitors, holograms, virtual or augmented reality models or $3 \mathrm{D}$ printed models. Users are able to experience realistic $3 \mathrm{D}$ virtual elements with augmented depth perception and binocular field of view. Newer technology has now allowed for interaction with 3D objects within these virtual environments through the use of hand gestures or hand controllers. The authors describe our preliminary experience with $\mathrm{MxR}$ as an adjunct tool to traditional angiographic imaging in the preprocedural workup of patients with complex aneurysms using a novel spatial computer, the Magic Leap One device (Magic Leap, Inc Plantation, Florida) which uses a virtual retinal display, which superimposes 3D computer-generated imagery over real world objects, by projecting a digital light field into the user's eye.

Materials and Methods Tomographic Angiographic (DynaCT) data was imported and segmented to create $3 \mathrm{D}$ meshes of the intracranial vasculature. The $3 \mathrm{D}$ meshes were then projected into MxR space, allowing the operator to inspect the vasculature using a MxR headset (Magic Leap) as well as interact with the aneurysm and adjacent vessels (handling, rotation, magnification, and sectioning) using hand gestures or hand controllers.

Results 3D segmentation of a complex aneurysms was successfully performed and projected into MxR. Conventional and MxR visualization modes were equally effective in identifying and classifying the pathology. MxR visualization allowed the operators to manipulate the dataset to achieve a greater understanding of the anatomy of the parent vessel, the angioarchitecture of the aneurysm, and the surface contours of all visualized structures.

Conclusion This preliminary study demonstrates the feasibility of utilizing MxR for preprocedural evaluation in patients with anatomically complex neurovascular disorders. This novel visualization approach may serve as a valuable adjunct tool in deciding patient-specific management, including decisions on prognostication and open surgical and endovascular treatment options.

Disclosures G. Deib: None. D. Smith: None. B. Chaudhary: None. S. Boo: None. A. Tarabishy: None. J. Carpenter: None. A. Rai: None.

\section{E-196 THE RISK OF ISCHEMIA IN PREVENTIVE TEMPORARY ARTERY OCCLUSION OF THE CONTRALATERAL ANTERIOR CEREBRAL ARTERY DURING MICROSURGICAL CLIPPING OF ACOM ANEURYSMS}

${ }^{1} \mathrm{C}$ Wipplinger*, ${ }^{2} \mathrm{~F}$ Mrosk, ${ }^{1} S$ Tülü, ${ }^{1} \mathrm{C}$ Preuss-Hernandez, ${ }^{1} \mathrm{~W}$ Ho, ${ }^{1} \mathrm{~A}$ Görke, ${ }^{1} \mathrm{M}$ Ortler, ${ }^{1} \mathrm{O}$ Petr, ${ }^{1} \mathrm{C}$ Thomé. ${ }^{1}$ Department of Neurosurgery, Medical University of Innsbruck, Innsbruck, Austria; ${ }^{2}$ Department of Neurosurgery, Charité-Universitätsmedizin Berlin, Berlin, Germany

\subsection{6/neurintsurg-2020-SNIS.227}

Introduction Temporary artery occlusion (TAO) is commonly used to facilitate dissection and clipping of intracranial aneurysms. When clipping anterior communicating artery (Acom) aneurysms, a possible strategy is to perform early preventive TAO of the contralateral A1 segment of the anterior cerebral artery before dissecting towards the aneurysm. In case of intraoperative complications such as complicated aneurysm dome preparation or inadvertent rupture of the aneurysm, full proximal control can be quickly gained by additional TAO of the easily accessible ipsilateral A1. Also, right-sided approaches are sometimes preferred due to reduced risk of damage of the dominant hemisphere and right-handedness of the surgeon. However, this may lead to prolonged TAO times of the contralateral A1 and might involve the risk of cerebral ischemia, especially if the contralateral A1 is the dominant supplier. In the present study, we aimed to investigate the safety of this strategy.

Methods We retrospectively analyzed the clinical and imaging outcomes of Acom aneurysms treated at our institution over a period of seven years by a right-sided frontotemporal approach. Temporary contralateral A1 occlusion early after opening of the chiasmatic cistern was defined as early TAO. We evaluated the total TAO time, A1 dominance, intraoperative rupture rates as well as postoperative mRS. The primary outcome parameter was postoperative ischemia determined on CT scans within 48 hours after surgery by a board-certified neuroradiologist.

Results A total of 81 including 52 ruptured and 29 unruptured aneurysms were treated by microsurgical clipping over a period of seven years. In 48 patients (59\%) early contralateral A1 TAO was performed for an average of $22 \pm 20 \mathrm{~min}$ and the majority of these cases (30 patients) harbored a dominant contralateral A1. Bilateral TAO was performed in 22 patients $(27 \%)$ for $11 \pm 10 \mathrm{~min}$. Intraoperative rupture occurred in 20 patients $(25 \%)$ of which 10 received early TAO. Postoperatively, 5 patients showed postoperative ischemia (6\%). Bilateral TAO as well as dominant early TAO showed no significant correlation with ischemia or worse post-operative mRS. However, patients with ischemia had a significantly longer overall TAO time $(45 \pm 27$ mins) than patients without (19 \pm 18 mins) $(\mathrm{p}=0.02)$.

Conclusion In our patient population neither early TAO of the dominant A1 nor bilateral TAO appeared to have an increased risk for ischemia. However, prolonged TAO, regardless of which side should be avoided.

Disclosures C. Wipplinger: None. F. Mrosk: None. S. Tülü: None. C. Preuss-Hernandez: None. W. Ho: None. A. Görke: None. M. Ortler: None. O. Petr: None. C. Thomé: None. 